Check for updates

Cite this: Chem. Sci., 2018, 9, 8975

๑ All publication charges for this article have been paid for by the Royal Society of Chemistry

\section{Highly efficient room-temperature phosphorescence and afterglow luminescence from common organic fluorophores in 2D hybrid perovskites $\dagger$}

\author{
Shuming Yang, ${ }^{a}$ Duobin Wu, ${ }^{a}$ Wenjing Gong, ${ }^{a}$ Qiuqin Huang, ${ }^{a}$ Hongyu Zhen, ${ }^{b}{ }^{b}$ \\ Qidan Ling (D)*b and Zhenghuan Lin (D)*ab
}

Regardless of rapid development of organic room-temperature phosphorescence (RTP) originating from phosphors in crystals, highly efficient and persistent RTP from common fluorophores is very rare. Herein, 1,8-naphthalimide (NI), a common organic fluorophore, is doped into organic cations of 2D layered organic/inorganic hybrid perovskites (OIHPs) to yield thin films and powders with yellow RTP of NI in air. The triplet excitons of $\mathrm{NI}$ are mainly derived from Wannier excitons of inorganic perovskite through energy transfer (ET) for films, and from singlet excitons of $\mathrm{NI}$ through intersystem crossing (ISC) for powder. Consequently, the quantum yield $\left(\Phi_{\mathrm{P}}\right)$, lifetime $(\tau)$ and color of RTP can be tuned by changing the fluorophore and halide in the perovskites, as well as their solid morphology. A white emission, comprising the blue one from the perovskite and yellow RTP $\left(\Phi_{\mathrm{P}}=25.6 \%, \tau=6.3 \mathrm{~ms}\right)$ from $\mathrm{NI}$, is obtained in $\mathrm{Br}$-based OlHPs in powder. Cl-based OlHPs exhibit fluorescence/phosphorescence dual emission in thin films, and yellow afterglow phosphorescence in powders $\left(\Phi_{\mathrm{P}}=56.1 \%, \tau=35 \mathrm{~ms}\right)$. The unique performance of the OIHPs with RTP can make them widely applicable in the field of information technology as security ink, and white and afterglow LEDs as single luminescent materials.
Received 10th August 2018 Accepted 25th September 2018

DOI: $10.1039 / \mathrm{c} 8 \mathrm{sc03563f}$

rsc.li/chemical-science halogen bonding and Van der Waals force), which is helpful to fix molecular conformation and to limit the spreading of oxygen and water. ${ }^{4}$ Additionally, organic phosphors as guests, physically or chemically doped in various hosts (such as layered double hydroxides, metal-organic frameworks, the cavity of macrocycles, steroidal compounds, polymers, and supermolecular gel), ${ }^{5}$ would yield RTP for the well-designed hostguest interactions. Despite these amazing works, it is a huge challenge to develop a simple strategy for constructing highly efficient and persistent RTP materials based on fluorophores, especially thin films, which can be widely applied in various fields by solution-processable methods.

Organic-inorganic hybrid perovskites (OIHPs), combining organic and inorganic semiconductors at the molecular level, have received considerable attention recently due to their advantages of both high carrier mobility and low-temperature solution processing. ${ }^{6} 2 \mathrm{D}$ layered OIHPs with a natural quantum well structure can easily form uniform and dense films, and consequently display good thermal stability and moisture resistance. ${ }^{7}$ Besides, the rigid inorganic laminate of perovskites is favorable to produce phosphorescence of the organic part by controlling the energy transfer from Wannier excitons to triplet excitons. For example, Koshimizu's group reported a series of 2naphthylmethyl amine-based OIHPs $\left(\mathrm{C}_{10} \mathrm{H}_{7} \mathrm{CH}_{2} \mathrm{NH}_{3}\right)_{2} \mathrm{PbBr}_{x} \mathrm{I}_{4-x}$ exhibiting a phosphorescence emission at $10 \mathrm{~K} .{ }^{8}$ Papavassiliou
${ }^{a}$ Fujian Key Laboratory of Polymer Materials, Fujian Normal University, Fuzhou 350007, China. E-mail: zhlin@fjnu.edu.cn

${ }^{b}$ College of Chemistry and Materials Science, Fujian Normal University, Fuzhou 350007, China.E-mail: qdling@fjnu.edu.cn

$\dagger$ Electronic supplementary information (ESI) available: Experimental details for the synthesis, characterization, and LED device fabrication, powder XRD patterns, transient luminescence decays, emission spectra. See DOI: 10.1039/c8sc03563f 
and Ema successively observed RTP phenomenon with a naked eye from layered OIHPs employing 1-naphthylmethyl amine as the organic cation. ${ }^{9}$ However, these phosphorescence emissions at room temperature are too weak to be used in various fields.

Here, we present a simple design strategy to produce highly efficient and persistent RTP from OIHPs by doping fluorophores into the organic part, which can effectively inhibit the triplet-triplet annihilation (TTA) process (Fig. 1). 1,8-Naphthalimide (NI) is a kind of common fluorescent dye. So far, there have been no reports of its RTP in an air atmosphere, although Br-substituted NI at the 4-position obtained RTP for a heavy atom effect induced by $\mathrm{Br}^{5 \boldsymbol{b}, \boldsymbol{g}, 10}$ When NI introduced into Br-based OIHPs (PEPB-NIA) and Cl-based OIHPs (PEPC-NIA), a white emission of PEPB-NIA including the blue one from perovskite and yellow one from NI (RTP, $\Phi_{\mathrm{P}}=25.6 \%$ ) was realized through optimizing the content of NIAB; a yellow RTP was obtained from PEPC-NIA with the a $\Phi_{\mathrm{P}}$ of $56.1 \%$ under an air atmosphere. To the best of our knowledge, the $\Phi_{\mathrm{P}}$ of PEPCNIA is the highest value of reported yellow RTP in air. Replacing NI with other fluorophores can also form OIHPs with highly efficient and persistent RTP in air.

\section{Results and discussion}

\section{Bromine-based perovskite thin films}

Firstly, 1,8-naphthalimide-based ammonium bromide (NIAB), obtained from 1,8-naphthalic anhydride (Scheme S1 $\dagger$ ), was doped into the organic ammonium, phenylethylammonium bromide (PEAB), to form 2D hybrid perovskite film PEPB-NIA with $\mathrm{PbBr}_{2}$ by a solution spin-coating method (Fig. 1). A series of thin films PEPB-NIA5, PEPB-NIA10, PEPB-NIA15, PEPB-NIA22 and PEPB-NIA30, where the last one or two digits denotes the feed content of NIAB in organic cations (the same below), have been synthesized. A non-doped perovskite film PEPB was also synthesized for purposes of comparison. There is only a (00l) diffraction peak found in their X-ray diffraction (XRD) plots, which confirm the formation of highly oriented 2D perovskite planes parallel to the glass surface (Fig. S1†). PEPB-NIA shows almost identical diffraction peaks with $\mathrm{PEPB}$, which indicate that NIAB was successfully introduced into the laminate of perovskites. The diffraction peaks of PEPB-NIA30 become blunt, thus a doping content of NIAB above $30 \%$ would destroy the $2 \mathrm{D}$ layered structure of the perovskite.

The photos of PEPB-NIAs under UV light and their photoluminescence spectra are shown in Fig. 2a. The luminescence color of PEPB-NIA films gradually changes from deep blue to yellow, when the doping content of NIAB increases from 0 to $30 \%$. This is ascribed to a new low-energy band appearing at around $554 \mathrm{~nm}$ with two shoulder peaks at about $600 \mathrm{~nm}$ and $656 \mathrm{~nm}$. The perovskite film without NIAB (i.e. PEPB) only exhibits a sharp high-energy peak at $407 \mathrm{~nm}$ which originates from the free excitons of the inorganic part of the perovskite. ${ }^{11}$ With the increasing amount of NIAB, the intensity of the blue emission becomes weak, while that of the yellow emission becomes strong. It seems that down-conversion from $407 \mathrm{~nm}$ to $554 \mathrm{~nm}$ takes place. However, the low-energy emission caused by NIAB is very different from that of the NIAB film at room temperature (Fig. 2b), but similar to that of NI in toluene at $77 \mathrm{~K} .^{10}$ As a result, it can be inferred that the band at $554 \mathrm{~nm}$ should be assigned to phosphorescence emission, and that it
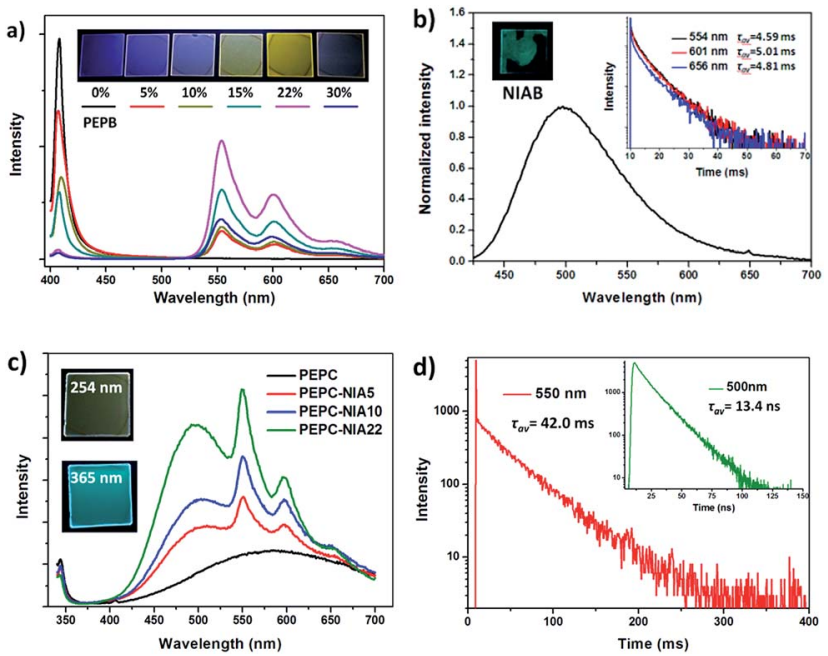

Fig. 2 Photos under UV light and the photoluminescence spectra of the PEPB-NIA film with different contents of NIAB (a) excited at $395 \mathrm{~nm}$, and NIAB (b) and PEPC-NIAC (c) films excited at $365 \mathrm{~nm}$ and $330 \mathrm{~nm}$, respectively. Transient luminescence decays of PEPB-NIA22 (b, inset) and PEPC-NIA22 (d) films measured at different emission peaks.

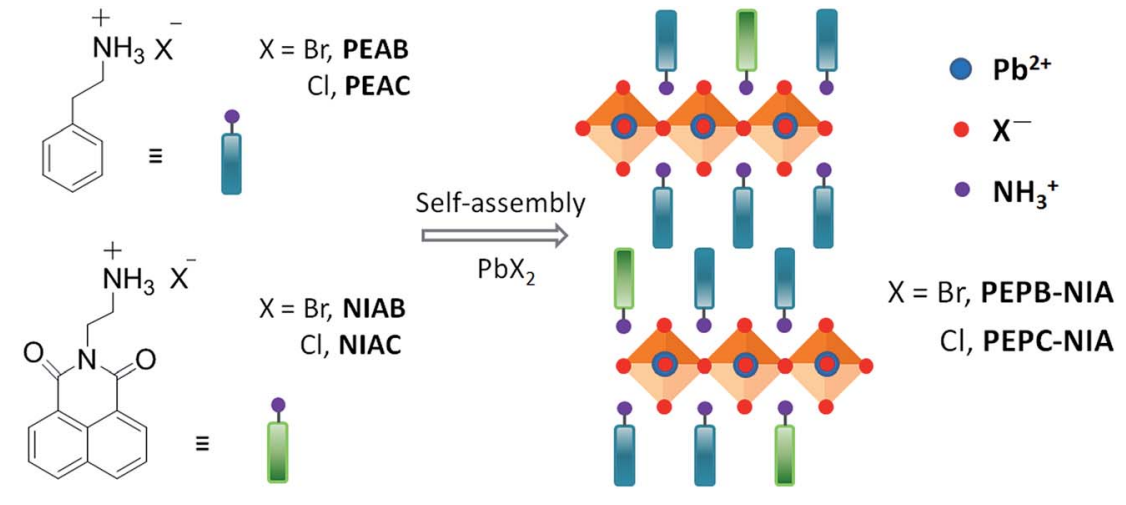

Fig. 1 Synthesis and structural illustration of the doped perovskites. 
originated from the triplet excited states of single molecular NI. The rigid laminate and lattice of the perovskite can prohibit the rotation and vibration of NI molecules, and limit access of oxygen, which contribute to the radiative decay of its triplet excitons, consequently resulting in the bright RTP.

The RTP of the films can be confirmed by the transient luminescence properties of PEPB-NIA5 and PEPB-NIA22. Their time-resolved transient luminescence decays at $554 \mathrm{~nm}, 600 \mathrm{~nm}$ and $656 \mathrm{~nm}$ are plotted in Fig. $2 \mathrm{~b}$ (inset) and S2. $\dagger$ All of the decay curves at different wavelengths are recorded using three discrete exponentials with excellent fits and give a similar average life time in the range of 4.59-5.94 ms (Table S1 $\dagger$ ). The quantum yield $\left(\Phi_{\mathrm{P}}\right)$ of the phosphorescence $(530-700 \mathrm{~nm})$ of these films was measured and is shown in Fig. S3. $\dagger$ At first the $\Phi_{\mathrm{P}}$ gradually rises with the increasing amount of NIAB, reaching $11.4 \%$ at PEPB-NIA22. However, the $\Phi_{\mathrm{P}}$ of the film decreases to $6.5 \%$, when $30 \%$ of NIAB was doped into the PEPB. The initial enhancement in quantum yield should be attributed to the effective energy transfer between the inorganic perovskite and NI. The decline of the $\Phi_{\mathrm{P}}$ of PEPB-NIA30 is caused by the poor quality of the crystal (Fig. S1†).

The absorption spectra of PEPB, NIAB and PEPB-NIA22 films (Fig. S4 $\dagger$ ) were collected to make clear the main origin of phosphorescence. PEPB shows two strong absorption peaks at $296 \mathrm{~nm}$ and $402 \mathrm{~nm}$, while NIAB mainly displays a band centred at $340 \mathrm{~nm}$. A combination of absorption of PEPB and NIAB results in a broadband absorption of PEPB-NIA22 from $250 \mathrm{~nm}$ to $430 \mathrm{~nm}$. Consequently, there should be two possible channels to produce the triplet excitons of NIAB in the perovskite film (Fig. 3a): (i) excitation of the perovskite followed by tripletstate energy transfer $\left({ }^{3} \mathrm{ET}\right) ;{ }^{8,9 b}$ (ii) excitation of NIAB followed by singlet-state energy transfer $\left({ }^{1} \mathrm{ET}\right)$ and ${ }^{3} \mathrm{ET}$. However, PEPBNIA22 shows consistent excitation spectra when monitored at $554 \mathrm{~nm}, 600 \mathrm{~nm}$ and $650 \mathrm{~nm}$ (Fig. S5†), which are similar to that of the inorganic part of the perovskite monitored at $417 \mathrm{~nm}$. It indicates that the phosphorescence emission of the organic part mainly results from Wannier excitons of the inorganic perovskite (Channel 1). Ema and co-workers verified that the triplet-triplet Dexter-type energy transfer from Wannier excitons to the triplet state of the organic part is very effective, and that the back transfer from the organic part does not occur, due to quite fast relaxation in the fluorophore vibronic band. ${ }^{g b}$

\section{Chlorine-based perovskite thin films}

To further verify the mechanism of luminescence of perovskite films, bromine is replaced by chlorine to obtain PEPC-NIA film series (Fig. 1), also exhibiting characteristic PXRD patterns of 2D perovskite films (Fig. S6†). The absorption and emission spectra of PEPC-NIA5, PEPC-NIA10 and PEPC-NIA22, as well as a non-doped perovskite film PEPC, is shown in Fig. S7 $\dagger$ and 2c. PEPC exhibits a sharp excitonic absorption band from $320 \mathrm{~nm}$ to $350 \mathrm{~nm}$. Upon excitation at $330 \mathrm{~nm}$, PEPC exhibits a narrow and a wide emission peak located at $344 \mathrm{~nm}$ and $585 \mathrm{~nm}$, respectively. Like reported broadband white-light emitting perovskites, ${ }^{12}$ the two bands originate from the free excitons and self-trapped excitons, respectively. ${ }^{13}$ Due to the very weak

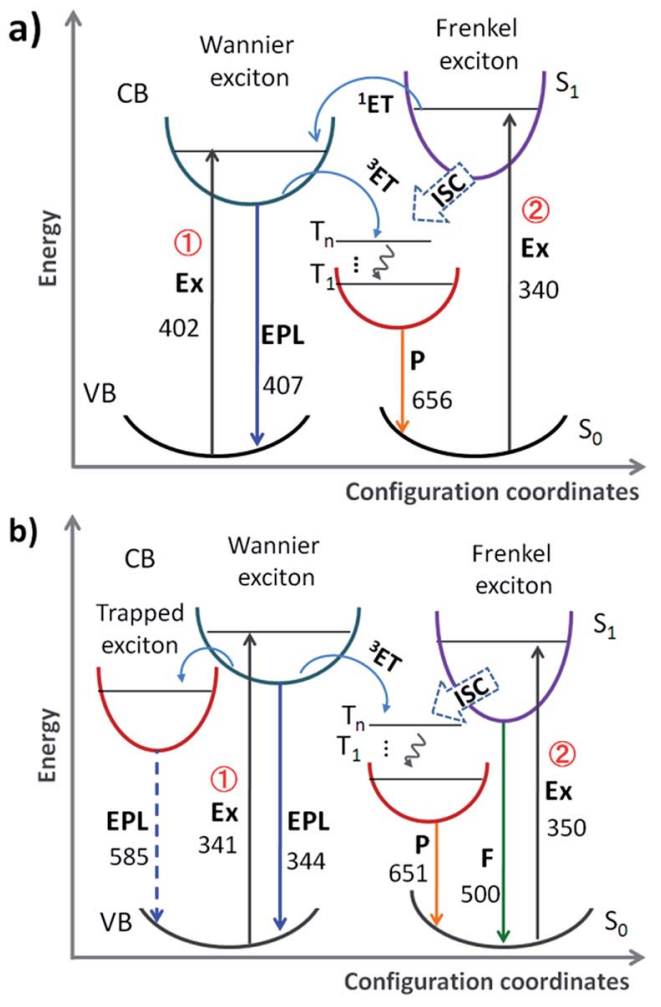

Fig. 3 Schematics summarizing the main possible energy transfer (ET) in PEPB-NIA (a) and PEPC-NIA (b) films: excitation (Ex), excitonic photoluminescence (EPL), phosphorescence (P), and fluorescence (F). The unit of the numbers is $\mathrm{nm}$. ISC mainly occurs in powder samples.

absorption of the NIAC film, PEPC-NIA films show similar absorption spectra to that of PEPC. However, PEPC-NIA films show very different emission spectra from that of the PEPC film. Although the narrow emission band remains at $344 \mathrm{~nm}$, the broad emitting peak of PEPC-NIAs is blue shifted from $585 \mathrm{~nm}$ to $500 \mathrm{~nm}$, accompanied by three shoulder peaks at $550 \mathrm{~nm}, 597$ $\mathrm{nm}$ and $651 \mathrm{~nm}$, corresponding well to the phosphorescence emission of NI. ${ }^{10}$ The average lifetime of the three shoulder peaks of PEPC-NIA22 is calculated to be about $41 \mathrm{~ms}$ (Table S2 $\dagger$ ) from their time-resolved transient luminescence decays (Fig. 2d and $\mathrm{S} 8 \dagger$ ). The lifetime of PEPC-NIA22 at $500 \mathrm{~nm}$ is $13.43 \mathrm{~ns}$, which should originate mainly from organic singlet excitons of NIA, and slightly from the trapped excitons of the perovskite.

Interestingly, PEPC-NIAs display excitation-wavelengthdependent photoluminescence properties. Taking PEPC-NIA22 as an example, its thin film shows dark yellow light and bright blue-green light under $254 \mathrm{~nm}$ and $365 \mathrm{~nm}$ UV lamps, respectively (Fig. 2c, inset). The luminescence spectra of the PEPCNIA22 film excited at different wavelengths were measured and are shown in Fig. S9. $\dagger$ Under the excitation of $340 \mathrm{~nm}$ (i.e. the maximum absorption wavelength of PEPC, Fig. S7 $\dagger$ ), the intensity of phosphorescence emission at $550 \mathrm{~nm}$ is obviously stronger than that of the fluorescence one at $500 \mathrm{~nm}$. However, with the increasing excitation wavelength, the peak at $550 \mathrm{~nm}$ becomes weak and disappears at $365 \mathrm{~nm}$ excitation, while the band centred at $500 \mathrm{~nm}$ becomes stronger. This suggests that the excitation of the inorganic perovskite is favorable to the 
phosphorescence emission. The result confirms that the Channel 1 RTP mechanism of PEPB-NIA (Fig. 3a) is also effective for PEPC-NIA films.

However, unlike PEPB-NIA, PEPC-NIA gives both fluorescence and phosphorescence under the excitation of a wavelength less than $350 \mathrm{~nm}$. When excited at a wavelength more than $350 \mathrm{~nm}$, PEPC-NIA would mainly yield fluorescence emission at $500 \mathrm{~nm}$. This should be because the ${ }^{1} \mathrm{ET}$ in Channel 2 is invalid in the PEPC-NIA system due to the higher exciton energy level of the inorganic perovskite (Fig. 3b). As a result, the excitation of NIAC would obtain fluorescence. Another difference of luminescence between PEPC-NIA and PEPB-NIA is that the intensity of the narrow excitonic emission of the inorganic part in PEPC-NIA at about $340 \mathrm{~nm}$ has little change with the increasing content of the doped organic cation (Fig. 2c). It should be attributed to the existence of a self-trapped state in the inorganic lattice (Fig. 3b). ${ }^{12}$ In the case of PEPC without NIAC, most Wannier excitons spontaneously transfer to the trapped state to yield a weak and wide emission band. When doping NIAC into perovskites, the triplet state of NIAC competes with the trapped state and shares the Wannier excitons through ${ }^{3} \mathrm{ET}$ (Channel 1). As a result, the increases of the doping amount of NIAC improve the intensity of phosphorescence, but do not change much the intensity of the excitonic emission of the inorganic part of the perovskite. Just because of the existence of trapped state, the efficiency of energy transfer from Wannier excitons to triplet excitons in PEPC-NIAs is lower than that in PEPB-NIAs, which results in a low luminescence quantum yield (4.21\%) of PEPC-NIA22.

\section{Applications of perovskites as ink}

Due to the excitation-wavelength-dependent photoluminescence properties of PEPC-NIA22, it can be employed as security ink to realize the encryption and decryption of data. For example, four letters 'FJNU' were first handwritten on a glass sheet by using PEPC-NIA22 as ink, and then twelve other letters were written by using NIAB as ink to form four words 'FOOD', 'Jack', 'Nice' and 'Urge', and to complete the encrypting process for their same emitting color under a $365 \mathrm{~nm}$ UV lamp (Fig. 4a). However, 'FJNU' can be recognized for exhibiting a different emission color from other letters upon $254 \mathrm{~nm}$ UV irradiation. The glass substrate was substituted by paper to expand the application of PEPC-NIA22 as security ink. Interestingly, the ink on the paper shows a very different luminescence color in the oxygen and nitrogen atmosphere, and persistent luminescence in the nitrogen ambient. For instance, the Chinese word of phosphorescence written on the paper emits sky-blue light (only fluorescence) in air due to the quenching of yellow phosphorescence of PEPC-NIA by oxygen permeating into paper, but white light including fluorescence and RTP in $\mathrm{N}_{2}$, under a $365 \mathrm{~nm}$ UV lamp. When the UV lamp is turned off, the word in the $\mathrm{N}_{2}$ atmosphere displays a yellow light (only phosphorescence) for several seconds (Fig. 4b). Three kinds of luminescent states can be switched reversibly by changing ambient and turning on/off the UV lamp. The emission color of the Chinese word remains unchanged after switching the atmosphere between $\mathrm{O}_{2}$ and $\mathrm{N}_{2}$ ten times (Fig. S10 $\dagger$ ). Consequently, PEPCNIAs employed as security ink can be applied in the field of anticounterfeit technology. Taking a name card as an example, four letters 'FJNU' were written at in the top right corner of the name card (Fig. 4c). The letters are invisible under daylight in air, but visible in blue light under a UV lamp. When the card was put into a chamber filled with $\mathrm{N}_{2}$, the letters 'FJNU' emitting white light were recognized under a $365 \mathrm{~nm}$ UV lamp. After turning off the lamp, they give a yellow afterglow emission.

\section{Perovskite powders}

The unique properties of PEPB-NIA and PEPC-NIA thin films encourage us to synthesize their powder counterparts by precipitation in acetone. PEPB-NIA5, PEPB-NIA10 and PEPBNIA20 powders also exhibit two emission bands that originated from free excitons of the perovskite (before $525 \mathrm{~nm}$ ) and triplet excitons (after $525 \mathrm{~nm}$ ) of NI (Fig. 5a), like their films. The emission intensity of free excitons of perovskite powders gradually decreases with the amount of NIA, but is higher than that of the corresponding thin films (Fig. 2a). This indicates that ${ }^{3} \mathrm{ET}$ between Wannier excitons and triplet excitons of NI in powder is not effective as in films. However, the $\Phi_{\mathrm{P}}$ of powders is summarized in Table 1 is 2-3 times higher than that of films, reaching $25.6 \%$. This indicates that ET between Wannier excitons and triplet excitons of NIA is not the main reason which caused the high $\Phi_{\mathrm{P}}$ of PEPB-NIAs. The improvement of $\Phi_{\mathrm{P}}$
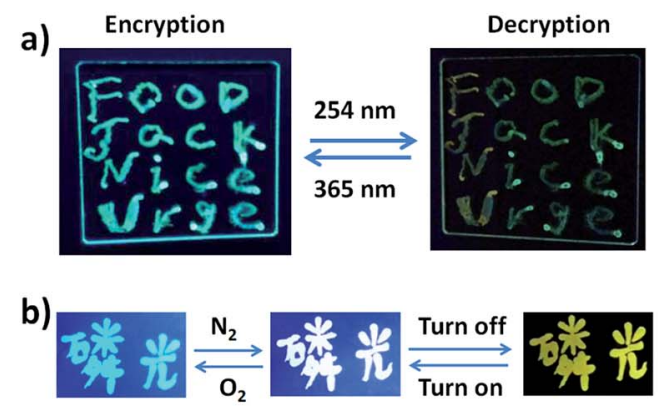
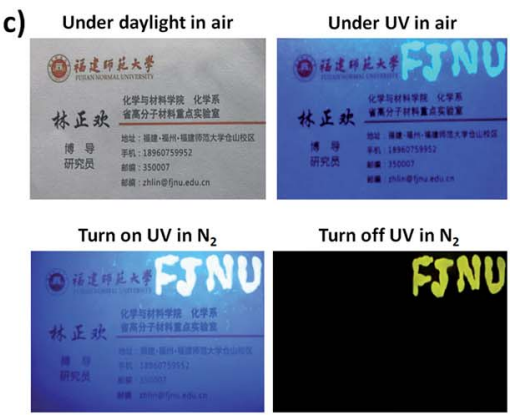

Fig. 4 Application of PEPC-NIA22 as security ink: (a) encryption and decryption of 'FJNU' hidden in four words under $254 \mathrm{~nm}$ and $365 \mathrm{~nm}$ of UV light. (b) Different luminescent states of the Chinese word of phosphorescence on paper. (c) An illustration of anti-counterfeit technology by writing the letters 'FJNU' on a name card by hand. 

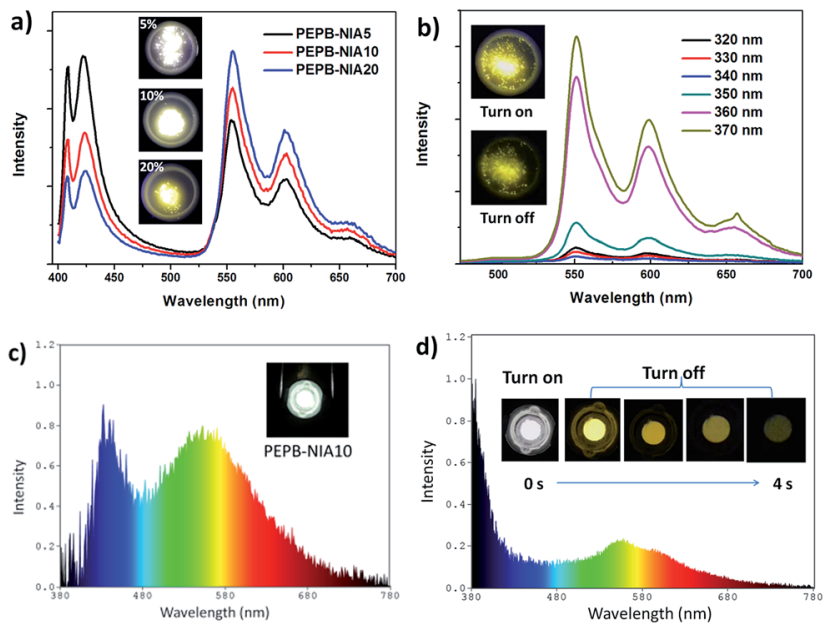

Fig. 5 Photos under UV light and the photoluminescence spectra of PEPB-NIA (a) excited at $395 \mathrm{~nm}$ and PEPC-NIA5 (b) powders excited at different wavelengths. Photos under UV light and the photoluminescence spectra of LEDs based on PEPB-NIA10 (c) and PEPCNIA5 (d) powders.

should be attributed to the tighter molecular packing and more complete lattice in powder than in films, which can produce the heavy atom effect of halogens, consequently resulting in ISC taking place in PEPB-NIA powders (Fig. 3a). PXRD patterns of PEPB-NIA powders were measured to confirm the difference of the packing structure between powder and film samples. Weak diffraction peaks at $25.5^{\circ}, 28.5^{\circ}$ and $30.8^{\circ}$, corresponding to the short contact of $3.5 \AA$, $3.13 \AA$ and $2.9 \AA$, exist in the PXRD pattern of PEPB-NIA powders (Fig. S11 $\dagger$ ). This indicates that the intermolecular distance is shorter in powders than in films, which provides a possibility of the heavy atom effect from halogen bonding between NI and Br. Due to the comparative intensity of the two bands, PEPB-NIA5, PEPB-NIA10 and PEPB-NIA20 powders display a white emission with a CIE of $(0.34,0.30)$, $(0.39,0.36)$ and $(0.42,0.40)$.

Cl-based perovskite powders display greatly different luminescence properties from their films. Taking PEPC-NIA5 as an example, the powder mainly shows a phosphorescent emission band from $525 \mathrm{~nm}$ to $700 \mathrm{~nm}$, whatever the excitation wavelength (Fig. 5b). A yellow afterglow was found after turning off the UV light of $365 \mathrm{~nm}$. When excited at $340 \mathrm{~nm}$ which can excite both inorganic and organic parts of the perovskite,
PEPC-NIA5 powder gives a low quantum yield of $2.4 \%$ similar to its film for the existence of the trapping state. Surprisingly, the $\Phi_{\mathrm{P}}$ of PEPC-NIA5 powder reaches $56.1 \%$ upon $370 \mathrm{~nm}$ of excitation which cannot excite the inorganic perovskite. There are plenty of weak diffraction peaks at $27.5-33.6^{\circ}$ corresponding to the short contact of 3.2-2.66 $\AA$ in the PXRD pattern of PEPCNIA5 powders (Fig. S11†). A short intermolecular distance makes it possible to form halogen bonding between NI and $\mathrm{Cl}$. Consequently, the highly efficient phosphorescence of PEPCNIA powders should come from the singlet state excitons through the ISC process caused by the heavy atom effect, like PEPB-NIA powder, although the heavy atom effect of $\mathrm{Cl}$ is inferior to that of Br. Through analyzing reported single crystal data of PEAC and PEAB, ${ }^{11,14}$ it is found that the volume of a unit cell of PEAC (2091.85 $\AA$ ) is smaller than that of PEAB (2268 $)$ ), which indicates that Cl-based perovskites tend to form a more compact crystal structure for small $\mathrm{Cl}$ atoms. The tight packing of Cl-based perovskites should be responsible for the heavy atom effect, and high $\Phi_{\mathrm{P}}$ and persistent RTP of PEPC-NIA5 powder. Fig. S12-S15 $\dagger$ show the transient luminescence decay curves of PEPB-NIA and PEPC-NIA powders at the main emission peaks with the lifetime summarized in Table 1.

\section{White and afterglow LEDs}

The perovskite PEPB-NIA powders can be used as single luminescent materials applied in UV-driven white white-light emitting devices (UV-WLEDs) due to their broad emission when excited by 355-385 $\mathrm{nm}$. Powder samples were deposited on a commercially available ultraviolet (UV) LED which is based on the AlGaN material system and emits around $380 \mathrm{~nm}$ (Fig. S16 $\dagger$ ). The loading amount of perovskite powders was controlled in the range of $30 \%$. Electroluminescence spectra of the UV-WLEDs based on PEPB-NIA under $350 \mathrm{~mA}$ of current are displayed in Fig. 5c, S17 and S18 $\uparrow$ with the data summarized in Table 1. All the electroluminescence spectra cover the whole visible region from $380 \mathrm{~nm}$ to $750 \mathrm{~nm}$, consequently producing a white emission. PEPB-NIA10 gives a relatively pure white emission with a CIE of $(0.32,0.36)$, which is close to that of pure white light of $(0.33,0.33)$. The CCT and CRI values of the UVWLED based on PEPB-NIA can be tuned from $5030 \mathrm{~K}$ to 12483 $\mathrm{K}$, and from 73 to 87 .

Afterglow LEDs can continue emitting light for a while after turning off the power at night, which gives us a chance to adapt to darkness. The powder PEPC-NIA5 can be used as

Table 1 Photophysical data for PEPB-NIA and PEPC-NIA powder

\begin{tabular}{|c|c|c|c|c|c|c|c|}
\hline \multirow[b]{2}{*}{ Powder } & \multicolumn{3}{|c|}{ Photoluminescence $^{a}$} & \multicolumn{4}{|c|}{ Electroluminescence } \\
\hline & $\lambda_{\mathrm{em}}(\mathrm{nm})$ & $\tau_{\mathrm{av}}(\mathrm{ms})$ & $\Phi_{\mathrm{P}}(\%)$ & $\lambda_{\mathrm{em}}(\mathrm{nm})$ & CIE & CCT (K) & CRI \\
\hline PEPB-NIA10 & 555 & 6.26 & 18.7 & 433,533 & $(0.32,0.36)$ & 6090 & 76 \\
\hline PEPB-NIA20 & 555 & 6.27 & 25.6 & 440,558 & $(0.35,0.40)$ & 5030 & 73 \\
\hline PEPC-NIA5 & 550 & 35.1 & 56.1 & 385,539 & $(0.34,0.37)$ & 5322 & 75 \\
\hline
\end{tabular}

${ }^{a}$ Excited at $395 \mathrm{~nm}$ for PEPB-NIA, and $370 \mathrm{~nm}$ for PEPC-NIA. 
a luminescent material to fabricate afterglow LEDs due to its long lifetime. Fig. 5d shows the electroluminescence spectra and pictures of a PEPC-NIA5-based LED. Due to the weak absorption of PEPC-NIA5 for bands after $380 \mathrm{~nm}$, the LED also displays a white light for the combination of emission of the chip and PEPC-NIA5. When the electricity is turned off, the LED can emit yellow phosphorescence for several seconds. The afterglow time of PEPC-NIA5 in the LED extends to several seconds. It can be explained by the reason that PEPC-NIA is further fixed by encapsulated silica gel through hydrogen bonding between the carbonyl group of NI and hydroxyl group of silica, which can inhibit non-radiative relaxation and quenching pathways of excitons. ${ }^{15}$

\section{Perovskites based on other fluorophores}

To confirm the universality of the strategy producing the phosphorescence of single molecules, other ammonium salts 1-NTAB, 2-NOAB and NAAC (Fig. 6a) replacing NIAB and NIAC as luminophors were doped into PEAB or PEAC to form OIHP thin film PEPB-NTA15 and PEPB-NOA15, and powder PEPBNTA20, PEPB-NOA20 and PEPC-NAA5, respectively (Scheme $\mathrm{S} 2 \dagger$ ). For the purpose of comparison, PEPB-NTA100 and PEPBNOA100 films containing $100 \%$ of $1-\mathrm{NTAB}$ and $2-\mathrm{NOAB}$ (without PEAB) were prepared under the same conditions. The PEPBNTA100 film shows weak orange phosphorescence $\left(\Phi_{\mathrm{P}}=0.4 \%\right)$, while PEPB-NOA100 has no phosphorescence emission (Fig. 6b), which are consistent with the reported results. ${ }^{9}$ The lifetime of emission of PEPB-NTA100 at $575 \mathrm{~nm}, 615 \mathrm{~nm}$ and $650 \mathrm{~nm}$ is measured to be $175 \mu \mathrm{s}, 362 \mu \mathrm{s}$ and $462 \mu \mathrm{s}$, respectively (Fig. S19 and Table S3†). After being doped into PEAB, both kinds of luminophors emit bright phosphorescence in OIHP films in air (Fig. 6b). The phosphorescence emission of PEPBNTA15 locates at $473 \mathrm{~nm}, 507 \mathrm{~nm}$ and $550 \mathrm{~nm}$ with a lifetime of about $3.5 \mathrm{~ms}$, while PEPB-NOA15 shows a green RTP with 4.86
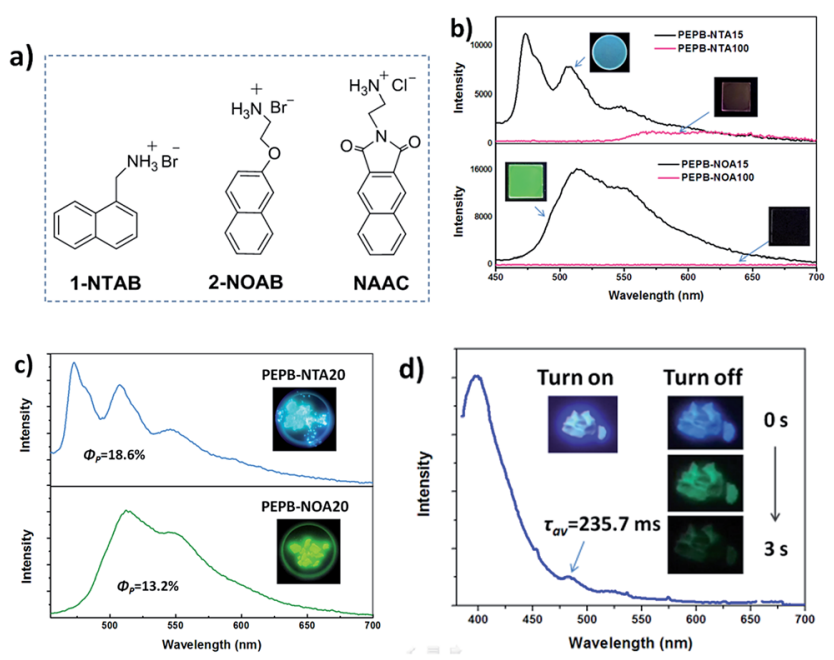

Fig. 6 Structure of fluorophore-based ammonium salts (a). Photos under UV light and the photoluminescence spectra of PEPB-NTA15 and PEPB-NOA15 films (b), PEPB-NTA20 and PEPB-NOA20 powders (c), and PEPC-NAA5 powder (d) excited at $365 \mathrm{~nm}, 395 \mathrm{~nm}$ and 370 $\mathrm{nm}$, respectively. $\mathrm{ms}, 4.47 \mathrm{~ms}$ and $6.13 \mathrm{~ms}$ of lifetime at $514 \mathrm{~nm}, 544 \mathrm{~nm}$ and $595 \mathrm{~nm}$, respectively (Fig. S20 and Table S3†). The $\Phi_{\mathrm{P}}$ of PEPBNTA15 and PEPB-NOA15 is measured to be $2.24 \%$ and $4.4 \%$, respectively. In the case of powders, PEPB-NTA20 and PEPBNOA20 give higher $\Phi_{\mathrm{P}}$ of $18.6 \%$ and $13.2 \%$ with the lifetime located in the range between $3.95 \mathrm{~ms}$ and $8.84 \mathrm{~ms}$ (Fig. S21, Table S4 $\dagger$ ). PEPC-NAA5 exhibits both fluorescence at $399 \mathrm{~nm}$ $\left(\tau_{\mathrm{av}}=5.33 \mathrm{~ns}\right)$ and phosphorescence at $482 \mathrm{~nm}\left(\tau_{\mathrm{av}}=235.7 \mathrm{~ms}\right)$ with a total quantum yield of $25.5 \%$ (Fig. S22, Table S5†). We note that a relevant perovskite study ${ }^{\mathbf{1 6}}$ showing an outstanding phosphorescence yield of $11.2 \%$ has been published online just before submitting this paper. It also confirms the perovskite incorporated with fluorophores is a superior system for RTP.

\section{Conclusions}

In summary, an effective and universal strategy is presented in this work to construct highly efficient and persistent RTP materials by doping common fluorophores (such as naphthalimide and naphthalene) into 2D OIHPs. Due to the homogeneous dispersion in organic host cations, the RTP comes from the triplet states of the unimolecular fluorophores. The triplet excitons are mainly obtained from triplet state ET of Wannier excitons and ISC of the singlet excited state. The RTP can be tailored by the type of halogen atom and fluorophore, and morphology of OIHPs. In films, PEPB-NIAs with a $\mathrm{Br}$ atom give a strong phosphorescence emission of NI, while PEPC-NIAs with a $\mathrm{Cl}$ atom display dual emission of fluorescence and phosphorescence of NI, and excitation-wavelength-dependent luminescence properties. In powders, the $\Phi_{\mathrm{P}}$ of both PEPB-NIA and PEPC-NIA is improved 3-10 times, relative to that of their films. Lastly, NI is replaced by other fluorophores to give blue RTP and green persistent RTP $(\tau=0.24 \mathrm{~s})$ in air. This indicates that doping fluorophores into OIHPs is a universal strategy of designing highly efficient RTP materials.

\section{Conflicts of interest}

There are no conflicts to declare.

\section{Acknowledgements}

This work was supported by the National Natural Science Foundation of China (21374017 and 21574021), the Natural Science Foundation of Fujian Province (2017J01684), and the Educational Commission of Fujian Province (JA14068).

\section{Notes and references}

1 (a) Y. Han, Y. You, Y.-M. Lee and W. Nam, Adv. Mater., 2012, 24, 2748-2754; (b) J. Cao, X. Ma, M. Min, T. Cao, S. Wu and H. Tian, Chem. Commun., 2014, 50, 3224-3226; (c) Q. Zhao, C. Huang and F. Li, Chem. Soc. Rev., 2011, 40, 2508-2524; (d) G. Zhang, G. M. Palmer, M. W. Dewhirst and C. L. Fraser, Nat. Mater., 2009, 8, 747-751; (e) M. A. Baldo, D. F. O'Brien, Y. You, A. Shoustikov, S. Sibley, 
M. E. Thompson and S. R. Forrest, Nature, 1998, 395, 151154.

2 F.-Q. Han, C.-M. Han and H. Xu, Chin. Chem. Lett., 2016, 27, 1193-1200.

3 (a) J. Wei, B. Liang, R. Duan, Z. Cheng, C. Li, T. Zhou, Y. Yi and Y. Wang, Angew. Chem., Int. Ed., 2016, 55, 1558915593; (b) Z. Yang, Z. Mao, X. Zhang, D. Ou, Y. Mu, Y. Zhang, C. Zhao, S. Liu, Z. Chi, J. Xu, Y.-C. Wu, P.-Y. Lu, A. Lien and M. R. Bryce, Angew. Chem., Int. Ed., 2016, 55, 2181-2185; (c) W. Zhao, Z. He, J. W. Y. Lam, Q. Peng, H. Ma, Z. Shuai, G. Bai, J. Hao and B. Z. Tang, Chem, 2016, 1, 592-602; (d) B. Xu, H. Wu, J. Chen, Z. Yang, Z. Yang, Y.-C. Wu, Y. Zhang, C. Jin, P.-Y. Lu, Z. Chi, S. Liu, J. Xu and M. Aldred, Chem. Sci., 2017, 8, 1909-1914; (e) O. Bolton, K. Lee, H.-J. Kim, K. Y. Lin and J. Kim, Nat. Chem., 2011, 3, 205-210.

4 O. Bolton, D. Lee, J. Jung and J. Kim, Chem. Mater., 2014, 26, 6644-6649.

5 (a) S. Hirata, K. Totani, J. Zhang, T. Yamashita, H. Kaji, S. R. Marder, T. Watanabe and C. Adachi, Adv. Funct. Mater., 2013, 23, 3386-3397; (b) H. Chen, X. Yao, X. Ma and H. Tian, Adv. Opt. Mater., 2016, 4, 1397-1401; (c) X. Yang and D. Yan, Adv. Opt. Mater., 2016, 4, 897-905; (d) M. S. Kwon, D. Lee, S. Seo, J. Jung and J. Kim, Angew. Chem., Int. Ed., 2014, 53, 11177-11181; (e) R. Gao and D. Yan, Chem. Sci., 2017, 8, 590-599; (f) X. Yang and D. Yan, Chem. Sci., 2016, 7, 4519-4526; (g) D. Li, F. Lu, J. Wang, W. Hu, X.-M. Cao, X. Ma and H. Tian, J. Am. Chem. Soc., 2018, 140, 1916-1923; (h) M. S. Kwon, Y. Yu, C. Coburn, A. W. Phillips, K. Chung, A. Shanker, J. Jung, G. Kim, K. Pipe, S. R. Forrest, J. H. Youk, J. Gierschner and J. Kim, Nat. Commun., 2015, 6, 8947.

6 (a) B. Saparov and D. B. Mitzi, Chem. Rev., 2016, 116, 45584596; (b) Z.-G. Zhou, Y. Zhao, C. Zhang, D. Zhou, Y. Chen, Z. Lin, H. Zhen and Q. Ling, J. Mater. Chem. A, 2017, 5, 6613-6621; (c) Z. Zhou, L. Huang, X. Mei, Y. Zhao, Z. Lin, H. Zhen and Q. Ling, Sol. Energy, 2016, 136, 210-216.
7 (a) S. A. Veldhuis, P. P. Boix, N. Yantara, M. Li, T. C. Sum, N. Mathews and S. G. Mhaisalkar, Adv. Mater., 2016, 28, 6804-6834; (b) N. Wang, L. Cheng, R. Ge, S. Zhang, Y. Miao, W. Zou, C. Yi, Y. Sun, Y. Cao, R. Yang, Y. Wei, Q. Guo, Y. Ke, M. Yu, Y. Jin, Y. Liu, Q. Ding, D. Di, L. Yang, G. Xing, H. Tian, C. Jin, F. Gao, R. H. Friend, J. Wang and W. Huang, Nat. Photonics, 2016, 10, 699-704.

8 N. Kawano, M. Koshimizu and K. Asai, J. Phys. Chem. C, 2012, 116, 22992-22995.

9 (a) G. C. Papavassiliou, G. A. Mousdis, G. Pagona, N. Karousis and M.-S. Vidali, J. Lumin., 2014, 149, 287-291; (b) K. Ema, M. Inomata, Y. Kato, H. Kunugita and M. Era, Phys. Rev. Lett., 2008, 100, 257401.

10 B. Ventura, A. Bertocco, D. Braga, L. Catalano, S. d'Agostino, F. Grepioni and P. Taddei, J. Phys. Chem. C, 2014, 118, 18646-18658.

11 N. Kawano, M. Koshimizu, Y. Sun, N. Yahaba, Y. Fujimoto, T. Yanagida and K. Asai, J. Phys. Chem. C, 2014, 118, 91019106.

12 S. Yang, Z. Lin, J. Wang, Y. Chen, Z. Liu, E. Yang, J. Zhang and Q. Ling, ACS Appl. Mater. Interfaces, 2018, 10, 1598015987.

13 E. R. Dohner, A. Jaffe, L. R. Bradshaw and H. I. Karunadasa, J. Am. Chem. Soc., 2014, 136, 13154-13157.

14 K. Thirumal, W. K. Chong, W. Xie, R. Ganguly, S. K. Muduli, M. Sherburne, M. Asta, S. Mhaisalkar, T. C. Sum, H. S. Soo and N. Mathews, Chem. Mater., 2017, 29, 3947-3953.

15 (a) X. Ma, C. Xu, J. Wang and H. Tian, Angew. Chem., Int. Ed., 2018, 57, 10854-10858; (b) S. Cai, H. Shi, Z. Zhang, X. Wang, H. Ma, N. Gan, Q. Wu, Z. Cheng, K. Ling, M. Gu, C. Ma, L. Gu, Z. An and W. Huang, Angew. Chem., Int. Ed., 2018, 57, 4005-4009.

16 H. Hu, F. Meier, D. Zhao, Y. Abe, Y. Gao, B. Chen, T. Salim, E. E. M. Chia, X. Qiao, C. Deibel and Y. M. Lam, Adv. Mater., 2018, 30, 1707621. 